\title{
Small angle $x$-ray scattering with a beryllium compound refractive lens as focusing optic
}

Andreas Timmann', Ralph Döhrmann, Tom Schubert, Horst Schulte-Schrepping, Ulrich Hahn, Marion Kuhlmann, Rainer Gehrke, Stephan Volkher Roth, Andreas Schropp, Christian Schroer, and Bruno Lengeler

Citation: Review of Scientific Instruments 80, 046103 (2009); doi: 10.1063/1.3104186

View online: http://dx.doi.org/10.1063/1.3104186

View Table of Contents: http://aip.scitation.org/toc/rsi/80/4

Published by the American Institute of Physics

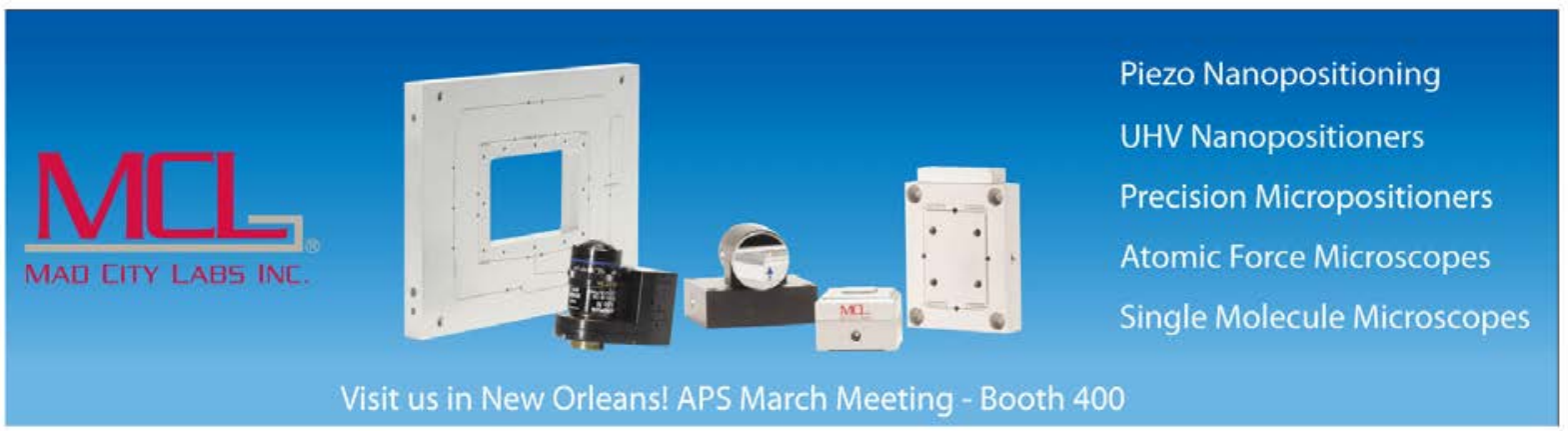




\title{
Small angle $\mathrm{x}$-ray scattering with a beryllium compound refractive lens as focusing optic
}

\author{
Andreas Timmann, ${ }^{1, a)}$ Ralph Döhrmann, ${ }^{1}$ Tom Schubert, ${ }^{1}$ Horst Schulte-Schrepping, ${ }^{1}$ \\ Ulrich Hahn, ${ }^{1}$ Marion Kuhlmann, ${ }^{1}$ Rainer Gehrke, ${ }^{1}$ Stephan Volkher Roth, \\ Andreas Schropp, ${ }^{2}$ Christian Schroer, ${ }^{2}$ and Bruno Lengeler ${ }^{3}$ \\ ${ }^{1}$ HASYLAB, DESY, Notkestrasse 85, 22607 Hamburg, Germany \\ ${ }^{2}$ TU Dresden, Zellescher Weg 16, 01069 Dresden, Germany \\ ${ }^{3}$ Physikalisches Institut IIB, RWTH Aachen, 52056 Aachen, Germany
}

(Received 9 December 2008; accepted 4 March 2009; published online 7 April 2009)

\begin{abstract}
At BW4 at HASYLAB a beryllium compound refractive lens (Be-CRL) is used for the focusing in small-angle x-ray scattering experiments. Using it provides the advantages of higher long-term stability and a much easier alignment compared to a setup with focusing mirrors. In our investigations presented here, we show the advantages of using a Be-CRL in small-angle and also ultra small-angle $\mathrm{x}$-ray scattering. We investigated the beam characteristics at the sample position with respect to spot size and photon flux. The spot size is comparable to that of a setup with focusing mirrors but with a gain in flux and better long-term stability. It is also shown that plane mirrors are still necessary to suppress higher order energies passing the monochromator. () 2009 American Institute of Physics. [DOI: 10.1063/1.3104186]
\end{abstract}

It is known that parabolic compound refractive lenses can be used to focus $\mathrm{x}$ rays. ${ }^{1,2}$ BW4 is a dedicated smallangle $\mathrm{x}$-ray scattering (SAXS) beamline located at a wiggler at DORIS III. A beryllium compound refractive lens (Be$\mathrm{CRL}$ ) is routinely used at this instrument to focus the $\mathrm{x}$-ray beam into a spot size of $65 \times 35 \mu \mathrm{m}^{2}$ and below in SAXS experiments. ${ }^{3}$ The beamline optics consist of a mirror for horizontal focusing, a Si-(111) double crystal monochromator, an aperture slit, and a vertical focusing mirror. Besides focusing these mirrors serve as low pass energy filters, removing higher harmonic radiation transmitted by the monochromator. ${ }^{4,5}$ In prospect of the micro- and nanofocus $\mathrm{x}$-ray scattering (MINAXS) beamline at the upcoming synchrotron light source PETRA III Be-CRLs were considered not only for focusing in SAXS but also in ultra small-angle $\mathrm{x}$-ray scattering (USAXS). The conventional option in USAXS at BW4 is focusing using mirrors. However, since long-term stability is a major issue for scanning and many in situ experiments, using a Be-CRL is considered as the better option. For this reason the beam characteristics of a Be-CRL had to be evaluated for SAXS and USAXS.

Therefore, we investigated the performance at BW4 at HASYLAB with an USAXS experimental setup. The complete setup is depicted in Fig. 1(a). For the experiment the focusing mirrors and the aperture slit were moved out of the beam path. A test chamber [Fig. 1(b)] was installed in the white beam at a distance of $28 \mathrm{~m}$ behind the wiggler, allowing one-to-one imaging of the source to the detector plane. It was equipped with two translation and two rotation stages for the alignment of the Be-CRL. The lens holder was water cooled to take away the heat produced by the white beam. The Be-CRL consisted of two parabolically shaped beryllium

\footnotetext{
${ }^{\text {a) }}$ Author to whom correspondence should be addressed. Electronic mail: andreas.timmann@desy.de.
}

lenses with a radius of curvature of $0.2 \mathrm{~mm}$ and a geometric aperture $2 R_{0}=0.9 \mathrm{~mm} .{ }^{1}$ The tilt absorber, which serves as a front end aperture, was opened to $1.6 \times 1.6 \mathrm{~mm}^{2}$. Hence the only optical elements remaining in the beam were the BeCRL, the $\mathrm{Si}(111)$ double crystal monochromator, and a guard slit. The photon energy was tuned to $E=8979 \mathrm{eV}$.

The sample was positioned $15 \mathrm{~m}$ behind the lens at the standard position for USAXS measurements at BW4

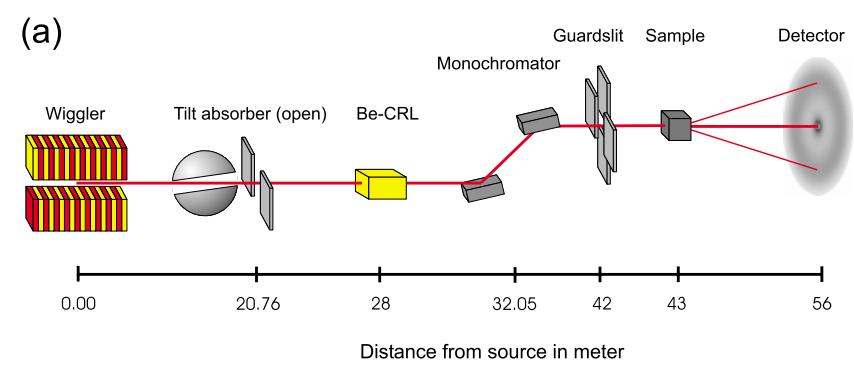

(b)

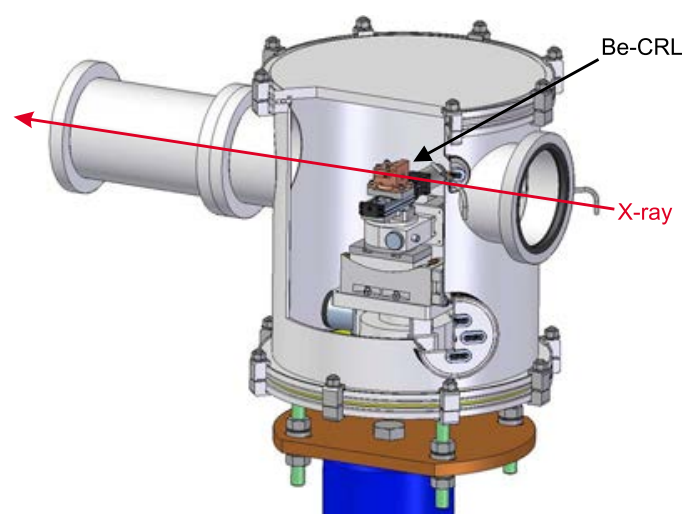

FIG. 1. (Color online) (a) Schematic BW4 setup for the Be-CRL investigation. (b) 3D computer model of the vacuum chamber for the refractive lens. 
(a)

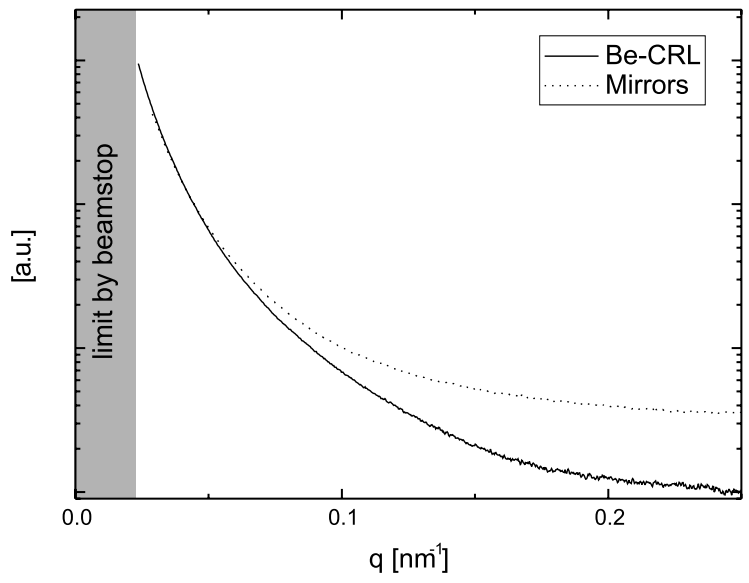

(b)

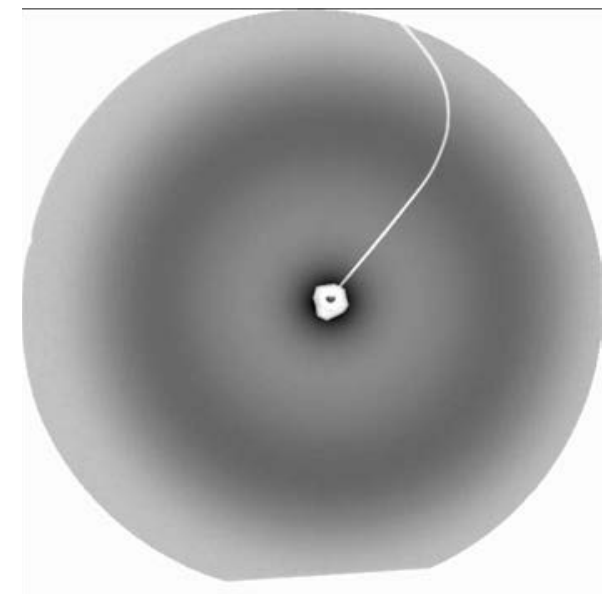

(c)

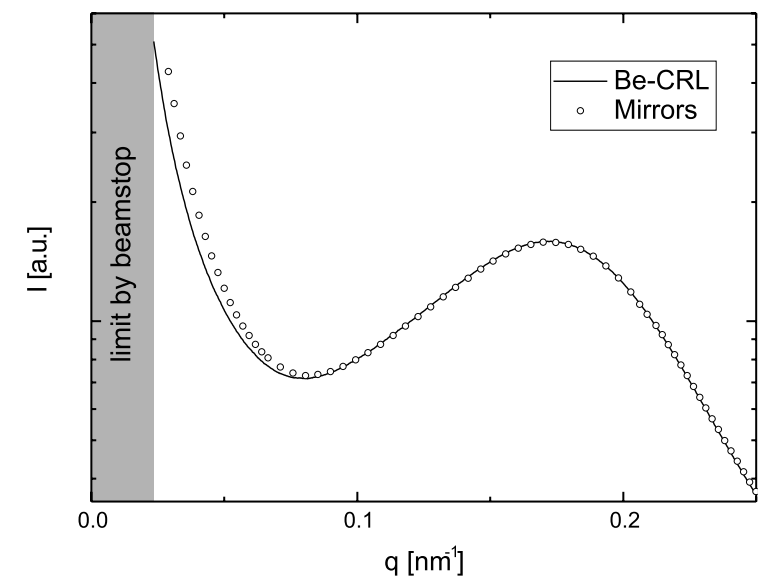

FIG. 2. (a) Background curve with the Be-CRL (straight line) and with mirrors (dotted line). (b) 2D scattering pattern of Lupolen (acquisition time: $2 \times 100 \mathrm{~s})$. The higher energy contribution is visible at the center of the beamstop. (c) Background corrected scattering curve averaged over an angle of $300^{\circ}$. A measurement with mirrors is shown for comparison (circles, acquisition time: $2 \times 150 \mathrm{~s}$ ). All curves are normalized for acquisition time and incoming flux.

[Fig. 1(a)]. The obtained background curve is shown in Fig. 2(a). For comparison a background curve with mirror optics is also shown. For $q<0.05 \mathrm{~nm}^{-1}$ the curves are identical. In the regime for $q>0.05 \mathrm{~nm}^{-1}$ the background with the Be-CRL is considerably lower than with mirror optics.

The photon flux was determined by a calibrated Lupolen standard sample of $1.5 \mathrm{~mm}$ thickness using the method described by Russell et al. ${ }^{6}$ A full pattern was recorded by a
marCCD165 detector placed $13 \mathrm{~m}$ behind the sample [Fig. 1(a)] and was afterward circularly averaged.

The resulting background corrected scattering pattern is shown in Fig. 2(b). There are no signs of diffraction peaks from the beamstop material. But in the middle of the beamstop the higher energies that penetrate through the stop are still visible. Here they only have to pass through a photodiode installed for intensity monitoring and a thin layer of lead behind it. This shows that when no mirrors are used for higher harmonic rejection, high energy photons are detected. A pair of plane mirrors in the optical path somewhere before the guard slits would remove the higher harmonic content of the spectrum. Decoupling focusing and higher harmonic rejection in this way is advantageous as it allows for a more flexible beamline.

The total photon flux was calculated to be 6.5 $\times 10^{10}$ photons $/ \mathrm{s} / \mathrm{mm}^{2}$ at $100 \mathrm{~mA}$ storage ring current. The flux observed in standard USAXS geometry with focusing mirrors is about $3 \times 10^{10}$ photons $/ \mathrm{s} / \mathrm{mm}^{2}$ at $100 \mathrm{~mA}$. Figure 2(c) shows the scattering curve of the Lupolen sample used in this experiment. It also shows the scattering curve of the same sample taken with mirrors as focusing optics. At scattering vectors $q<0.1 \mathrm{~nm}^{-1}$ the curve taken with the mirror optic shows a slightly higher diffuse scattering. Besides this, the obtained curves are identical.

The beam at sample position was investigated with high resolution two-dimensional (2D) position sensitive detector based on a thin scintillator that is imaged onto a charge-

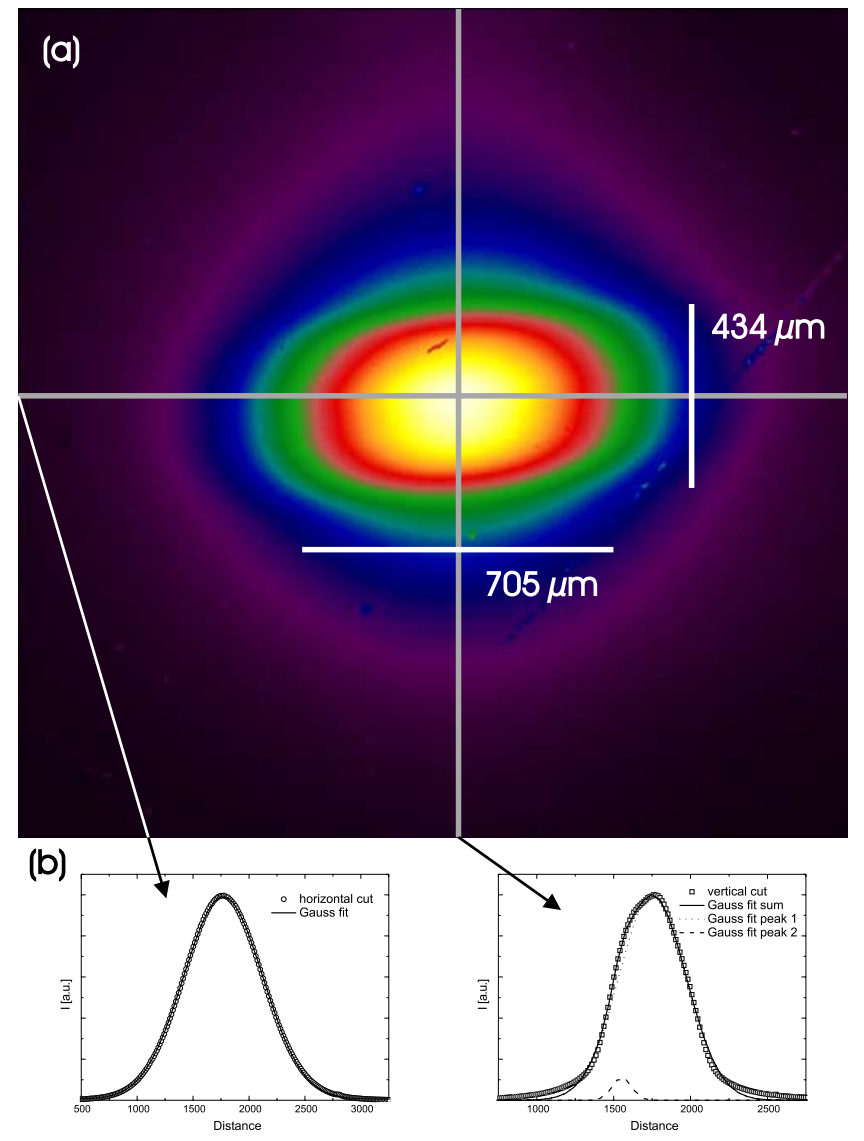

FIG. 3. (Color online) (a) Picture of the beam at sample position. The gray lines mark the positions of the cuts shown in the diagrams below. (b) Lefthorizontal cut with Gaussian fit. Right-vertical cut with fit of two Gaussian profiles. 
coupled device (CCD) chip by a visible light microscope optic. ${ }^{7,8}$ Figure. 3(a) shows the resulting image after $1 \mathrm{~s}$ accumulation time. From the first inspection it is seen that there are no large distortions in the beam geometry. The slight tilt is caused by the monochromator of BW4.

For the calculation of the actual beam size, horizontal and vertical sections were fitted with a Gaussian profile, as shown in Fig. 3(b). A Gaussian is expected in the converging beam behind the CRL in case of a homogeneous flat illumination of its aperture. For the horizontal cut we obtained a perfect match with a beam size of $705 \mu \mathrm{m}$ [full width at half maximum (FWHM)]. Due to the slight distortions caused by the monochromator, the vertical profile cannot be modeled by a simple Gaussian [Fig. 3(b) right]. Taking two profiles resulted in a nearly perfect match to the vertical beam geometry. The profile in the vertical direction has a width of $434 \mu \mathrm{m}$ (FWHM).

In the experiment described above we have shown that it is possible to benefit from the use of Be-CRL focusing optics not only in SAXS but also in USAXS geometry. Even though the major advantages are clearly in the SAXS region, there are no drawbacks of using them in USAXS. The beam profile shows no distortions caused by the lens, the background is considerably lower, and the overall photon flux was by a factor of 2 higher than in the mirrored setup. Although this gain was only achieved together with an increase in higher order contributions in the beam, which cannot be completely eliminated by detuning the second monochromator crystal, the advantage gained by using it outweighs this disadvantage. The higher harmonics problem can be easily solved by the use of plane mirrors to suppress the higher energies when using lenses for focusing. Then the flux is expected to be comparable in both scenarios but with the advantage of lower background, easier alignment, and higher long-term stability of the lens optics compared to focusing mirror optics. In the near future, the just described optical setup will be installed at the MINAXS beamline P03 at PETRA III/DESY fully exploiting these advantages.

${ }^{1}$ B. Lengeler, C. Schroer, J. Tümmler, B. Benner, M. Richwin, A. Snigirev, I. Snigireva, and M. Drakopoulos, J. Synchrotron Radiat. 6, 1153 (1999).

${ }^{2}$ B. Lengeler, C. Schroer, M. Kuhlmann, B. Benner, T. F. Günzler, O. Kurapova, F. Zontone, A. Snigirev, and I. Snigireva, J. Phys. D 38, A218 (2005).

${ }^{3}$ S. V. Roth, R. Döhrmann, M. Dommach, M. Kuhlmann, I. Kröger, R. Gehrke, H. Walter, C. Schroer, B. Lengeler, and P. Müller-Buschbaum, Rev. Sci. Instrum. 77, 085106 (2006).

${ }^{4}$ F. Gehrke, Rev. Sci. Instrum. 63, 455 (1992).

${ }^{5}$ R. Gehrke, M. Bark, D. Lewin, and S. Cunis, Rev. Sci. Instrum. 66, 1354 (1995).

${ }^{6}$ T. P. Russell, J. S. Lin, S. Spooner, and G. D. Wignall, J. Appl. Crystallogr. 21, 629 (1988).

${ }^{7}$ A. Koch, C. Raven, P. Spanne, and A. Snigirev, J. Opt. Soc. Am. A 15, 1940 (1998).

${ }^{8}$ J. Flügge, Diploma thesis, Technische Fachhochschule Wildau, Germany, 2004. 